\title{
OPEN Physical activity and the risk of abdominal aortic aneurysm: a systematic review and meta-analysis of prospective studies
}

\author{
Dagfinn Aune ${ }^{1,2,3 凶}$, Abhijit Sen ${ }^{4}$, Elsa Kobeissi ${ }^{5}$, Mark Hamer $^{6}$, Teresa Norat ${ }^{1}$ \& Elio Riboli ${ }^{1}$
}

The association between physical activity and risk of abdominal aortic aneurysm has been inconsistent with some studies reporting a reduced risk while others have found no association. We conducted a systematic review and meta-analysis of prospective studies to quantify the association. PubMed and Embase databases were searched up to 3 October 2020. Prospective studies were included if they reported adjusted relative risk (RR) estimates and $95 \%$ confidence intervals (CIs) of abdominal aortic aneurysm associated with physical activity. Summary RRs $(95 \% \mathrm{Cls})$ were estimated using a random effects model. Nine prospective studies (2073 cases, 409,732 participants) were included. The summary RR for high vs. low physical activity was $0.70\left(95 \% \mathrm{Cl}: 0.56-0.87,1^{2}=58 \%\right)$ and per 20 metabolic equivalent task (MET)-hours/week increase of activity was $0.84\left(95 \% \mathrm{Cl}: 0.74-0.95, \mathrm{I}^{2}=59 \%\right.$, $n=6)$. Although the test for nonlinearity was not significant $(p=0.09)$ the association appeared to be stronger when increasing the physical activity level from 0 to around 20-25 MET-hours/week than at higher levels. The current meta-analysis suggest that higher physical activity may reduce the risk of abdominal aortic aneurysm, however, further studies are needed to clarify the dose-response relationship between different subtypes and intensities of activity and abdominal aortic aneurysm risk.

Aortic aneurysms are dilatations of the aorta which when ruptured have an $80 \%$ overall mortality rate, with about one third dying before reaching hospital ${ }^{1}$ and $25-50 \%$ of cases undergoing surgery being fatal ${ }^{1-4}$. Some studies have shown improved survival rates in more recent years ${ }^{1}$. According to the Global Burden of Disease Study aortic aneurysms was estimated to account for 167,200 deaths and 3 million disability-adjusted life years in $2017^{5,6}$. The vast majority of aortic aneurysms are abdominal aortic aneurysms, with only $3 \%$ originating in the thorax ${ }^{7}$. In Caucasian populations the prevalence of abdominal aortic aneurysms is $4.7 \%{ }^{8-10}$, while it is $0.45 \%$ in Asian populations ${ }^{11}$. Established risk factors for abdominal aortic aneurysm include age ${ }^{12-14}$, male sex ${ }^{15}$, family history of abdominal aortic aneurysm ${ }^{16}$, low education ${ }^{15}$, hypertension ${ }^{13-15,17}$, elevated lipid levels ${ }^{16}$, coronary heart disease $\mathrm{e}^{12,15}$, peripheral artery disease ${ }^{12}$, chronic obstructive pulmonary disease and smoking ${ }^{13,15,18}$, while diabetes or diabetes medications appears to be protective ${ }^{12-16,19}$.

Physical activity has been shown to reduce blood pressure in randomized controlled trials ${ }^{20}$ and has been associated with reduced risk of hypertension in cohort studies ${ }^{21-24}$, and elevated blood pressure is a strong risk factor for abdominal aortic aneurysms ${ }^{17}$. Although there is strong evidence that physical activity reduces the risk of other vascular disorders such as coronary heart disease ${ }^{25}$, stroke ${ }^{25}$, and heart failure ${ }^{26}$, studies on physical activity and risk of abdominal aortic aneurysm have shown mixed results ${ }^{13,14,27-33}$. Some studies have shown inverse associations between higher physical activity and risk of abdominal aortic aneurysm ${ }^{27,29-33}$, however, other

\footnotetext{
${ }^{1}$ Department of Epidemiology and Biostatistics, School of Public Health, Imperial College London, St. Mary's Campus, Norfolk Place, Paddington, London W2 1PG, UK. '2Department of Nutrition, Bjørknes University College, Oslo, Norway. ${ }^{3}$ Department of Endocrinology, Morbid Obesity and Preventive Medicine, Oslo University Hospital, Oslo, Norway. ${ }^{4}$ Department of Public Health and Nursing, Faculty of Medicine and Health Sciences, Norwegian University of Science and Technology, Trondheim, Norway. ${ }^{5}$ Big Data Institute, University of Oxford, Oxford, UK. ${ }^{6}$ Institute Sport Exercise \& Health, Division Surgery \& Interventional Science, University College London, London, UK. ${ }^{\circledR}$ email: d.aune@imperial.ac.uk
} 
studies found no association ${ }^{13,14,28}$. The equivocal nature of these findings might be explained by relatively low numbers of events in some studies as abdominal aortic aneurysm are far less common than other cardiovascular events such as coronary heart disease and stroke.

Given the high mortality rates among patients with ruptured aortic aneurysm ${ }^{2}$, primary prevention is of major importance to reduce the public health burden of abdominal aortic aneurysms. We therefore conducted a systematic review and meta-analysis of prospective studies on physical activity and the risk of abdominal aortic aneurysm to clarify whether there is an association as well as the strength and shape of the dose-response relationship between the two.

\section{Results}

Out of a total of 9520 records retrieved by the search, 9473 were excluded based on title or abstract, and of the 47 publications that were assessed in more detail 9 cohort studies (2073 cases of abdominal aortic aneurysm

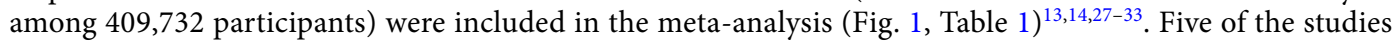
were from Europe and four studies were from the US. Three studies were identified from searches on other risk factors and abdominal aortic aneurysm ${ }^{27,28,31}$. Eight studies reported on physical activity and abdominal aortic aneurysm ${ }^{13,14,27,29-33}$ and one reported on aortic aneurysm overall ${ }^{28}$, and the latter study was still included in the overall analysis since most aortic aneurysms are abdominal aortic aneurysms (in a sensitivity analysis this study was excluded). Physical activity was assessed by self-reported (most studies) or interviewer-administered (one study) questionnaires, and two studies used validated questionnaires (Table 1). Three studies reported physical activity measures in METs ${ }^{14,31,32}$ two reported the duration of physical activity ${ }^{30,33}$, one reported physical activity on a scale from none to heavy ${ }^{27}$, one used a physical activity index ${ }^{28}$ and two simply compared no activity with any activity ${ }^{13,29}$. For three studies ${ }^{27,30,33}$ the physical activity measures were converted to MET-hours/week as described in the methods section.

The summary RR for individuals with high compared to low physical activity was 0.70 (95\% CI: $0.56-0.87$, $\mathrm{I}^{2}=58 \%$, pheterogeneity $=0.01$ ) (Fig. 2). There was no evidence of publication bias with Egger's test $(p=0.56)$ or with Begg's test $(p=0.60)$ or by inspection of the funnel plot (Supplementary Fig. 1). In sensitivity analyses excluding one study at a time the summary RR ranged from 0.66 (95\% CI: 0.53-0.83) when excluding the study by Wong et al. ${ }^{14}$ to $0.75(0.61-0.92)$ when excluding the study by Nordkvist et al. ${ }^{31}$ (Supplementary Table 2 ). Excluding one study (Honolulu Heart Program) reporting on total physical activity and aortic aneurysm in Japanese American men ${ }^{28}$ did not materially alter the results, and the summary RR was 0.67 (95\% CI: 0.54-0.83, $\left.\mathrm{I}^{2}=56 \%\right)$. Further exclusion of one additional study which reported on bicycling/walking ${ }^{30}$ left seven studies on leisure-time physical activity/exercise and aortic aneurysm ${ }^{13,14,27,29,31-33}$ did also not alter the results, and the summary RR was 0.68 (95\% CI: $\left.0.54-0.86, \mathrm{I}^{2}=61 \%\right)$.

Six studies were included in the dose-response analysis ${ }^{14,27,30-33}$ including 1641 cases and 377,637 participants. The summary RR per 20 MET-hours/week increase in physical activity was 0.84 (95\% CI: $\left.0.74-0.95, \mathrm{I}^{2}=59 \%\right)$ (Fig. 3a). There was no indication of publication bias with Egger's test $(p=0.71)$ or with Begg's test $(p=0.63)$. Sensitivity analyses excluding the Cancer Prevention Study $1^{27}$ (for which we approximated the physical activity level using data from the Cancer Prevention Study $2^{34}$ did not substantially alter the results and the summary RR was 0.86 (95\% CI: 0.77-0.97, $\mathrm{I}^{2}=53 \%$ ) (Supplementary Fig. 3). In influence analyses excluding one study at a time, the summary RR ranged from 0.80 (95\% CI: $0.73-0.88$ ) when excluding the study by Wong et al. ${ }^{14}$, to 0.86 (95\% CI: 0.77-0.97) when excluding the study by Hammond et al. ${ }^{27}$ (Supplementary Fig. 3). We also repeated the analysis of the highest versus lowest level of activity and risk of abdominal aortic aneurysm using the same studies that were included in the dose-response analysis and the summary RR was 0.69 (95\% CI: 0.54-0.88, $\left.\mathrm{I}^{2}=55 \%\right)$, which was very similar to the summary estimate among all studies.

Although the test for nonlinearity was not significant $(p=0.09)$ the association appeared to be stronger when increasing the physical activity level from 0 to around 20-25 MET-hours/week than at higher levels (Fig. 3b, Supplementary Table 2).

Subgroup and sensitivity analyses and study quality. Inverse associations were observed in most subgroup analyses defined by duration of follow-up, outcome type, outcome assessment, number of cases, study quality, and by whether studies excluded prevalent cases at baseline. The associations were often not significant when analyses were stratified by whether the studies adjusted for confounding factors or not, however, the number of studies in some subgroups were limited and there was no significant heterogeneity between any of the subgroup analyses (Table 2).

The mean (median) study quality scores were 6.8 (7.0) out of 9.0 points possible for the studies included in the analysis. The main reasons for a less than optimal study quality score were lack of representativeness of the general population $(n=3)$, lack of validation or lack of reporting of validation of physical activity questionnaires $(n=7)$, lack of exclusion or lack of reporting of exclusion of prevalent AAA cases at baseline $(n=3)$, and lack of reporting on loss to follow-up $(n=6)$ (Supplementary Table 3).

\section{Discussion}

This meta-analysis of 9 cohort studies including 2073 cases of abdominal aortic aneurysm among 409,732 participants suggest that a high level of physical activity reduces the risk of abdominal aortic aneurysms by $30 \%$. In the dose-response analysis there was a $16 \%$ reduction in the relative risk for each 20 MET-hours/week increase in physical activity (equivalent to approximately $2 \mathrm{~h} /$ week of vigorous running or bicycling, $3 \mathrm{~h} /$ week of moderate running/bicycling, or $6 \mathrm{~h}$ of brisk walking per week) and although the statistical test for nonlinearity was not significant, there was some indication of a stronger reduction in risk from 0 to 20-25 MET-hours/ week than at higher levels of activity. The inverse association was observed in European studies, but was not 


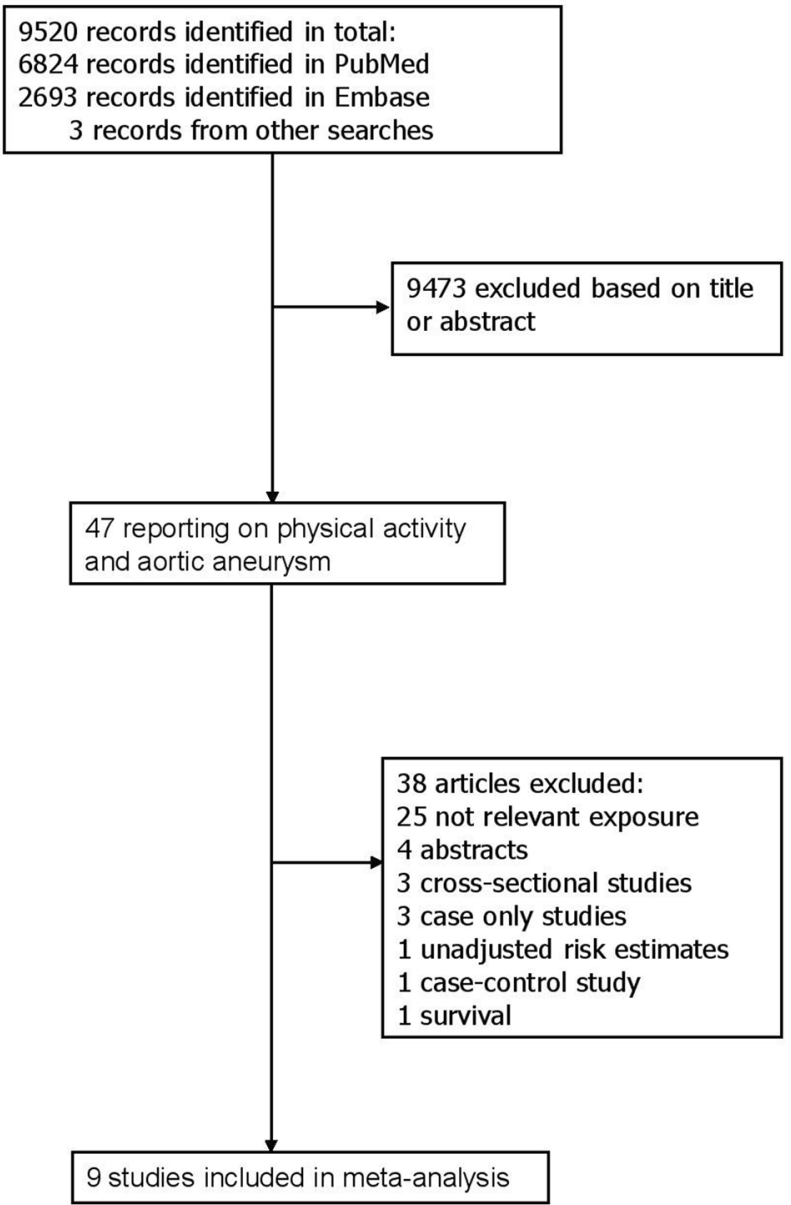

Figure 1. Flow-chart of study selection.

significant in American studies. The association was in the direction of reduced risk, but not always significant across subgroups, most likely because of the limited number of studies in several subgroups. However, there was no evidence of between subgroup heterogeneity with meta-regression analyses.

As with any meta-analysis of epidemiological studies this meta-analysis has limitations. There was moderately high heterogeneity in the analysis across studies. Different studies used different types of questionnaires to assess physical activity, which likely differed in levels of validity that may have contributed to part of the observed heterogeneity. All the included studies assessed physical activity by self-report, and in some studies the self-reported measures were validated. In addition, different studies differed in the way physical activity was reported from a qualitative description of the level of activity to a quantitative measure reported using MET-hours. This appears to be a recurring issue in physical activity epidemiology as we in several previous meta-analyses on physical activity and different health outcomes only were able to include a fraction of the available studies in the dose-response analyses, if at all ${ }^{35-37}$. In the current analysis we were able to include six out of nine studies in the dose-response analysis. It seems less likely that the three studies which were excluded from the dose-response analysis would have substantially altered the overall association as the summary estimate for the highest versus lowest analysis was very similar to the overall analysis when restricted to these same six studies. Any future studies should emphasize reporting the results in a way that can be combined with the available data, for example by reporting the level of physical activity in MET-hours per week and/or hours per week.

We conducted several subgroup analyses to investigate potential sources of heterogeneity, but found no evidence of heterogeneity between subgroups. The association was not significant in among studies with adjustment for BMI or obesity, but whether this is a chance finding, or is an indication that reduced adiposity may be a mechanism that explains part of the association between physical activity and abdominal aortic aneurysm needs further exploration in future studies with models with and without adjustment for adiposity in the same dataset. The non-significant associations among studies with adjustment for hypertension, blood pressure or lipids also needs to be interpreted carefully as these factors potentially could be mediating variables for the association between physical activity and abdominal aortic aneurysm. In addition, we cannot exclude the possibility that the association may have been partly confounded by tobacco smoking, which is a rather strong risk factor for abdominal aortic aneurysm ${ }^{18}$, because the association was not statistically significant when the analyses were restricted to the studies with adjustment for tobacco smoking. However, the association was still in the direction 


\begin{tabular}{|c|c|c|c|c|c|c|c|c|c|}
\hline $\begin{array}{l}\text { References, } \\
\text { country }\end{array}$ & $\begin{array}{l}\text { Study name or } \\
\text { description }\end{array}$ & Study period & $\begin{array}{l}\text { Number of } \\
\text { participants, } \\
\text { number of } \\
\text { cases }\end{array}$ & $\begin{array}{l}\text { Identification } \\
\text { of cases }\end{array}$ & $\begin{array}{l}\text { Physical } \\
\text { activity } \\
\text { assessment }\end{array}$ & $\begin{array}{l}\text { Type of activity, } \\
\text { subgroup }\end{array}$ & Comparison & $\begin{array}{l}\text { Relative } \\
\text { risk ( } 95 \% \\
\text { confidence } \\
\text { interval) }\end{array}$ & $\begin{array}{l}\text { Adjustment } \\
\text { for } \\
\text { confounders }\end{array}$ \\
\hline \multirow{3}{*}{$\begin{array}{l}\text { Oyenuga } \\
\text { et al. }{ }^{33} \text {, USA }\end{array}$} & \multirow{3}{*}{$\begin{array}{l}\text { The Atheroscle- } \\
\text { rosis Risk in } \\
\text { Communities } \\
\text { (ARIC) study }\end{array}$} & \multirow{3}{*}{$\begin{array}{l}\text { 1987-1989-2011, } \\
22.6 \text { years follow- } \\
\text { up }\end{array}$} & \multirow{3}{*}{$\begin{array}{l}14,375 \text { men and } \\
\text { women, age } \\
45-64 \text { years: } \\
545 \text { AAA cases }\end{array}$} & \multirow{3}{*}{\begin{tabular}{|l} 
Self-report \\
confirmed by \\
hospitalization \\
records and \\
death records, \\
linkage to hos- \\
pital records
\end{tabular}} & \multirow{3}{*}{$\begin{array}{l}\text { Interviewer } \\
\text { administered } \\
\text { Baecke ques- } \\
\text { tionnaire }\end{array}$} & \multirow{3}{*}{$\begin{array}{l}\text { Leisure-time } \\
\text { physical activity }\end{array}$} & $0 \mathrm{~min} / \mathrm{wk}$ & \multirow{3}{*}{$\begin{array}{l}1.00 \\
0.75(0.59-0.94) \\
0.78(0.64-0.95)\end{array}$} & \multirow[b]{3}{*}{ Age, sex, race } \\
\hline & & & & & & & \begin{tabular}{|l|}
$1-149$ \\
\end{tabular} & & \\
\hline & & & & & & & $\geq 150$ & & \\
\hline \multirow{14}{*}{$\begin{array}{l}\text { Hamer et al. }{ }^{32} \text {, } \\
\text { United King- } \\
\text { dom }\end{array}$} & \multirow{14}{*}{$\begin{array}{l}\text { The Health Sur- } \\
\text { vey for England } \\
\text { and the Scottish } \\
\text { Health Surveys }\end{array}$} & \multirow{14}{*}{$\begin{array}{l}1994,1995,1997 \\
1998,1999,2003 \\
2004,2006, \\
2008-2009 / 2011 \\
9.4 \text { years follow- } \\
\text { up }\end{array}$} & \multirow{14}{*}{$\begin{array}{l}65,093 \text { men and } \\
\text { women }(59,122 \\
\text { without preva- } \\
\text { lent CVD), } \\
\text { age } \geq 40 \text { years: } \\
113(76) \text { AAA } \\
\text { deaths }\end{array}$} & \multirow{14}{*}{$\begin{array}{l}\text { British National } \\
\text { Health Service } \\
\text { Central Registry }\end{array}$} & \multirow{14}{*}{$\begin{array}{l}\text { Validated ques- } \\
\text { tionnaire }\end{array}$} & \multirow{4}{*}{$\begin{array}{l}\text { Leisure-time } \\
\text { physical activ- } \\
\text { ity-meeting } \\
\text { recommenda- } \\
\text { tions }\end{array}$} & Inactive & 1.00 & \multirow{14}{*}{$\begin{array}{l}\text { Age, sex, } \\
\text { smoking, social } \\
\text { occupational } \\
\text { group, chronic } \\
\text { illnesses, } \\
\text { psychological } \\
\text { distress }\end{array}$} \\
\hline & & & & & & & Insufficient & $0.51(0.24-1.11)$ & \\
\hline & & & & & & & \begin{tabular}{|l|} 
Sufficient \\
\end{tabular} & $0.41(0.10-1.69)$ & \\
\hline & & & & & & & High & $0.77(0.24-2.45)$ & \\
\hline & & & & & & \multirow{10}{*}{$\begin{array}{l}\text { Leisure-time } \\
\text { physical activ- } \\
\text { ity, excluding } \\
\text { prevalent CVD } \\
\text { at baseline }\end{array}$} & $\begin{array}{l}<1.64 \text { MET- } \\
\text { hrs/wk }\end{array}$ & 1.00 & \\
\hline & & & & & & & \begin{tabular}{|l|}
$1.65-9.37$ \\
\end{tabular} & $0.91(0.55-1.52)$ & \\
\hline & & & & & & & $9.38-19.30$ & $1.29(0.75-2.20)$ & \\
\hline & & & & & & & \begin{tabular}{|l|}
$19.31-37.60$ \\
\end{tabular} & $0.85(0.45-1.61)$ & \\
\hline & & & & & & & $>37.60$ & $0.88(0.43-1.82)$ & \\
\hline & & & & & & & $\begin{array}{l}<1.64 \text { MET- } \\
\text { hrs/wk }\end{array}$ & 1.00 & \\
\hline & & & & & & & $1.65-9.37$ & $0.69(0.36-1.33)$ & \\
\hline & & & & & & & $9.38-19.30$ & $1.00(0.52-1.94)$ & \\
\hline & & & & & & & \begin{tabular}{|l|}
$19.31-37.60$ \\
\end{tabular} & $0.78(0.37-1.63)$ & \\
\hline & & & & & & & $>37.60$ & $0.86(0.37-1.96)$ & \\
\hline \multirow{5}{*}{$\begin{array}{l}\text { Nordkvist } \\
\text { et al. }{ }^{31} \text {, Sweden }\end{array}$} & \multirow{5}{*}{$\begin{array}{l}\text { Malmö Diet and } \\
\text { Cancer Study }\end{array}$} & \multirow{5}{*}{$\begin{array}{l}\text { 1991-1996-NA, } \\
20.7 \text { years follow- } \\
\text { up }\end{array}$} & \multirow{5}{*}{$\begin{array}{l}26,133 \text { men and } \\
\text { women, mean } \\
\text { age } 57.3 \text { years: } \\
353 \text { AAA cases }\end{array}$} & \multirow{5}{*}{$\begin{array}{l}\text { Linkage to } \\
\text { Inpatient and } \\
\text { Outpatient Reg- } \\
\text { ister, Cause of } \\
\text { Death Register }\end{array}$} & \multirow{5}{*}{ Questionnaire } & \multirow{5}{*}{$\begin{array}{l}\text { Leisure-time } \\
\text { physical activity }\end{array}$} & $\begin{array}{l}\text { 0-7.5 MET-hrs/ } \\
\text { wk }\end{array}$ & 1.00 & \multirow{5}{*}{ Age, sex } \\
\hline & & & & & & & \begin{tabular}{|l|}
$7.5-15$ \\
\end{tabular} & $0.72(0.35-1.04)$ & \\
\hline & & & & & & & $15-25$ & $0.50(0.35-0.72)$ & \\
\hline & & & & & & & $25-50$ & $0.54(0.39-0.74)$ & \\
\hline & & & & & & & $>50$ & $0.46(0.31-0.68)$ & \\
\hline \multirow[b]{4}{*}{$\begin{array}{l}\text { Stackelberg } \\
\text { et al. }{ }^{30} \text {, Sweden }\end{array}$} & & & & & & & Almost never & 1.00 & Age, education, \\
\hline & & & & & & & $<20 \mathrm{~min} /$ day & $0.83(0.53-1.32)$ & $\begin{array}{l}\text { smoking status, } \\
\text { pack-years, }\end{array}$ \\
\hline & & & & & & & $20-40$ & $0.72(0.45-1.16)$ & BMI, waist \\
\hline & $\begin{array}{l}\text { Cohort of Swed- } \\
\text { ish Men }\end{array}$ & $\begin{array}{l}\text { 1998-2011, } \\
13 \text { years follow- } \\
\text { up }\end{array}$ & $\begin{array}{l}14,249 \text { men, age } \\
\text { 65-75 years: } \\
156 \text { AAA cases }\end{array}$ & $\begin{array}{l}\text { Ultrasound } \\
\text { screening }\end{array}$ & $\begin{array}{l}\text { Validated ques- } \\
\text { tionnaire }\end{array}$ & $\begin{array}{l}\text { Walking, } \\
\text { bicycling }\end{array}$ & $\geq 40$ & $0.59(0.36-0.97)$ & $\begin{array}{l}\text { circumference, } \\
\text { healthy diet } \\
\text { score, alcohol, } \\
\text { diabetes melli- } \\
\text { tus, cardiovas- } \\
\text { cular disease, } \\
\text { hypertension, } \\
\text { hypercholester- } \\
\text { olemia }\end{array}$ \\
\hline & & & & & & & $\begin{array}{l}\text { 0.1-5.9 METs/ } \\
\text { wk }\end{array}$ & 1.00 & noking \\
\hline Wong et al. ${ }^{14}$ & Health Profes- & $1986-$ & 39,352 men, age & confirmed by & & & \begin{tabular}{|l|}
$6.0-13.7$ \\
\end{tabular} & $0.98(0.74-1.31)$ & hypertension, \\
\hline USA & $\begin{array}{l}\text { sionals Follow- } \\
\text { up Study }\end{array}$ & $\begin{array}{l}2002, \sim 14.6 \text { years } \\
\text { follow-up }\end{array}$ & $\begin{array}{l}\text { 40-75 years: } \\
\text { 376 AAA cases }\end{array}$ & $\begin{array}{l}\text { medical records, } \\
\text { National Death }\end{array}$ & Questionnaire & physical activity & $13.8-24.2$ & $1.15(0.85-1.56)$ & $\begin{array}{l}\text { diabetes, hyper- } \\
\text { cholesterolemia }\end{array}$ \\
\hline & & & & Index & & & $24.3-40.8$ & $0.95(0.67-1.35)$ & BMI \\
\hline & & & & & & & $\geq 40.9$ & $1.02(0.72-1.46)$ & \\
\hline $\begin{array}{l}\text { Lindblad } \\
\text { et al. }{ }^{29} \text {, Sweden }\end{array}$ & $\begin{array}{l}\text { Malmo Preven- } \\
\text { tive Study }\end{array}$ & $\begin{array}{l}\text { 1974-1991, } \\
21 \text { years follow- } \\
\text { up }\end{array}$ & \begin{tabular}{|l}
22,444 men, \\
mean age \\
43.7 years: \\
Nested case- \\
control study: \\
126 AAA cases \\
126 controls
\end{tabular} & $\begin{array}{l}\text { Hospital register } \\
\text { data, SwedVasc } \\
\text { quality control } \\
\text { data, death } \\
\text { certificates }\end{array}$ & Questionnaire & $\begin{array}{l}\text { Physical inac- } \\
\text { tivity }\end{array}$ & Yes vs. no & $2.67(1.42-5.01)$ & $\begin{array}{l}\text { Age, serum } \\
\text { triglycerides, } \\
\text { DBP, serum } \\
\text { cholesterol, } \\
\text { smoking }\end{array}$ \\
\hline $\begin{array}{l}\text { Tornwall et al. }{ }^{13} \text {, } \\
\text { Finland }\end{array}$ & $\begin{array}{l}\text { Alpha- } \\
\text { Tocopherol, } \\
\text { Beta-Carotene } \\
\text { Cancer Preven- } \\
\text { tion Study }\end{array}$ & $\begin{array}{l}1985-1988-1993 \\
5.8 \text { years follow- } \\
\text { up }\end{array}$ & $\begin{array}{l}29,133 \text { male } \\
\text { smokers, age } \\
\text { 50-69 years: } \\
181 \text { AAA cases }\end{array}$ & $\begin{array}{l}\text { National } \\
\text { hospital dis- } \\
\text { charge register, } \\
\text { national register } \\
\text { of causes of } \\
\text { death }\end{array}$ & Questionnaire & $\begin{array}{l}\text { Leisure-time } \\
\text { exercise }\end{array}$ & No vs. yes & $1.29(0.95-1.73)$ & $\begin{array}{l}\text { Age, cigarettes } \\
\text { per day, years } \\
\text { of smoking, } \\
\text { BMI, SBP, DBP, } \\
\text { total choles- } \\
\text { terol, HDL } \\
\text { cholesterol, } \\
\text { diabetes mel- } \\
\text { litus, education, } \\
\text { exercise, alpha- } \\
\text { tocopherol and } \\
\text { beta-carotene } \\
\text { supplementa- } \\
\text { tion group }\end{array}$ \\
\hline $\mathrm{C}$ & & & & & & & & & \\
\hline
\end{tabular}




\begin{tabular}{|c|c|c|c|c|c|c|c|c|c|}
\hline $\begin{array}{l}\text { References, } \\
\text { country }\end{array}$ & $\begin{array}{l}\text { Study name or } \\
\text { description }\end{array}$ & Study period & $\begin{array}{l}\text { Number of } \\
\text { participants, } \\
\text { number of } \\
\text { cases }\end{array}$ & $\begin{array}{l}\text { Identification } \\
\text { of cases }\end{array}$ & $\begin{array}{l}\text { Physical } \\
\text { activity } \\
\text { assessment }\end{array}$ & $\begin{array}{l}\text { Type of activity, } \\
\text { subgroup }\end{array}$ & Comparison & \begin{tabular}{|l|} 
Relative \\
risk $(95 \%$ \\
confidence \\
interval $)$
\end{tabular} & $\begin{array}{l}\text { Adjustment } \\
\text { for } \\
\text { confounders }\end{array}$ \\
\hline \multirow[b]{4}{*}{$\begin{array}{l}\text { Goldberg } \\
\text { et al. }{ }^{28}, \text { USA }\end{array}$} & \multirow[b]{4}{*}{$\begin{array}{l}\text { Honolulu Heart } \\
\text { Program }\end{array}$} & \multirow[b]{4}{*}{$\begin{array}{l}1965-1968-1988 \\
23 \text { years follow- } \\
\text { up }\end{array}$} & \multirow[b]{4}{*}{$\begin{array}{l}2710 \text { Japanese } \\
\text { American men, } \\
\text { age } 55-64 \text { years: } \\
119 \text { AA cases }\end{array}$} & \multirow[b]{4}{*}{$\begin{array}{l}\text { Medical, surgi- } \\
\text { cal and autopsy } \\
\text { records }\end{array}$} & \multirow[b]{4}{*}{$\begin{array}{l}\text { History of usual } \\
\text { 24-h physical } \\
\text { activity }\end{array}$} & \multirow[b]{4}{*}{$\begin{array}{l}\text { Physical activity } \\
\text { index }\end{array}$} & $\leq 29.6$ & 1.00 & \multirow{4}{*}{$\begin{array}{l}\text { Age, ventricular } \\
\text { rate, BMI, SBP, } \\
\text { serum choles- } \\
\text { terol, serum } \\
\text { triglycerides, } \\
\text { serum glucose, } \\
\text { serum uric acid, } \\
\text { hematocrit, } \\
\text { forced expira- } \\
\text { tory volume, } \\
\text { cigarettes per } \\
\text { day, alcohol }\end{array}$} \\
\hline & & & & & & & $29.7-32.1$ & $0.46(0.19-1.13)$ & \\
\hline & & & & & & & $3.22-35.5$ & $0.97(0.47-2.01)$ & \\
\hline & & & & & & & $\geq 35.6$ & $1.37(0.69-2.72)$ & \\
\hline \multirow{4}{*}{$\begin{array}{l}\text { Hammond } \\
\text { et al. }{ }^{27}, \text { USA }\end{array}$} & \multirow{4}{*}{$\begin{array}{l}\text { Cancer Preven- } \\
\text { tion Study } 1\end{array}$} & \multirow{4}{*}{$\begin{array}{l}\text { 1959-1960-NA, } \\
6 \text { years follow-up }\end{array}$} & \multirow{4}{*}{$\begin{array}{l}218,435 \text { men, } \\
\text { age } 50-69 \text { years: } \\
141 \text { AAA deaths }\end{array}$} & \multirow{4}{*}{$\begin{array}{l}\text { Linkage to } \\
\text { death records }\end{array}$} & \multirow{4}{*}{ Questionnaire } & \multirow{4}{*}{ Exercise } & Heavy & 1.00 & \multirow{4}{*}{ Age } \\
\hline & & & & & & & Moderate & $1.43(0.84-2.43)$ & \\
\hline & & & & & & & Slight & $1.87(1.13-3.10)$ & \\
\hline & & & & & & & None & $1.83(1.12-3.11)$ & \\
\hline
\end{tabular}

Table 1. Prospective studies of physical activity and abdominal aortic aneurysm. $A A A$ abdominal aortic aneurysm, $A A$ aortic aneurysm, $B M I$ body mass index, $D B P$ diastolic blood pressure, $H D L$ high-density lipoprotein, $S B P$ systolic blood pressure, $w k$ week.

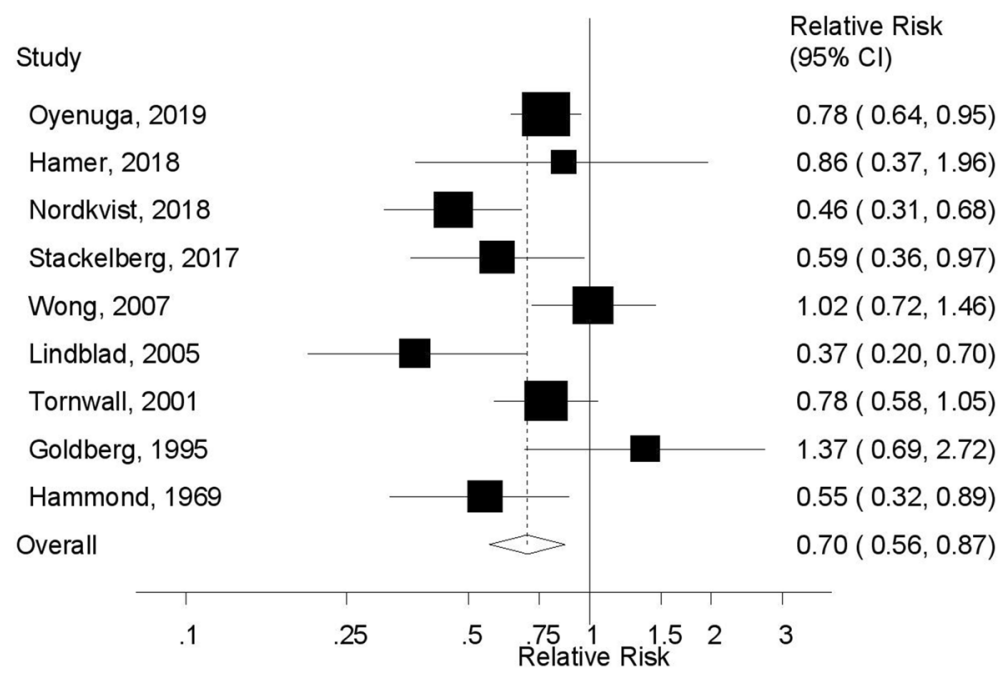

Figure 2. Physical activity and abdominal aortic aneurysm.

of reduced risk among studies with adjustment for smoking and since there was no significant heterogeneity between these subgroups with meta-regression analyses, limited statistical power may be another potential explanation. Any further studies should adjust more comprehensively for tobacco smoking and analyses stratified by smoking status may be needed to completely rule out the potential for residual confounding.

Reverse causation or a temporal bias where patients with prevalent abdominal aortic aneurysms before baseline may have reduced their physical activity level because of the condition could also have biased the results. However, the association persisted among the six studies that excluded participants with prevalent disease at baseline. Publication bias can affect meta-analyses of published studies. Although we found no evidence of publication bias in this analysis, it is possible that we may have had too low power to detect such bias, however, there was also no evidence of asymmetry in the funnel plots, which might argue against this being the case.

Little is known about the potential underlying mechanisms that could explain a beneficial effect of physical activity on the risk of abdominal aortic aneurysms. Physical activity has been associated with lower blood pressure and a lower risk of hypertension ${ }^{20-24}$, lower lipid levels ${ }^{38,39}$, as well as a lower risk of coronary heart disease $\mathrm{e}^{40}$, all of which are important risk factors for abdominal aortic aneurysms ${ }^{12,17,41}$. However, it is possible that the association is independent of traditional risk factors. A recent experimental study showed that low intensity exercise improved aortic wall structure and function in a mouse model of Marfan syndrome, while these benefits were attenuated at higher intensity exercise ${ }^{42}$. Reduced expression of matrix metalloproteinases 2 and 9 explained the reduced elastin fragmentation ${ }^{42}$. Whether these findings can be transferred to humans and individuals without Marfan syndrome is unclear and whether other mechanistic pathways are involved needs further study.

The current meta-analysis has several strengths including the relatively large sample size, comprehensive subgroup and sensitivity analyses, detailed linear and nonlinear dose-response analyses and moderately high 
A Physical activity and abdominal aortic aneurysm, per 20 MET-hours/week

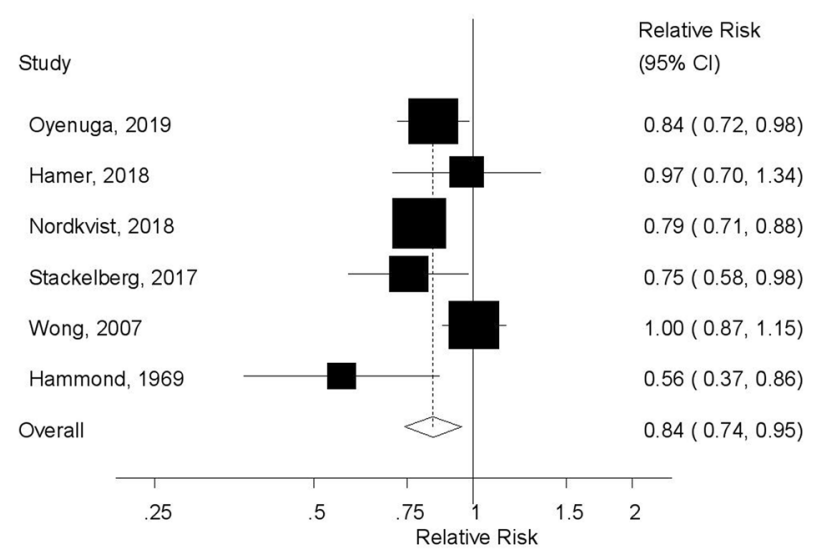

$\mathbf{B}$ Physical activity and abdominal aortic aneurysm, nonlinear dose-response

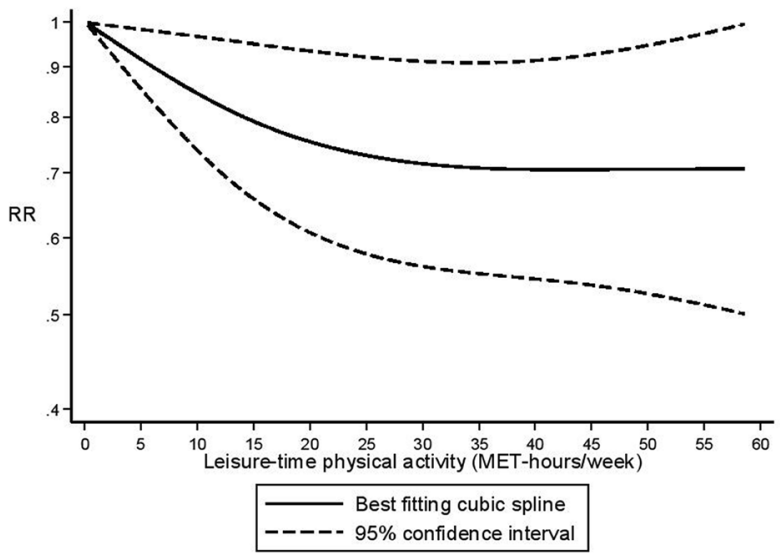

Figure 3. Physical activity and abdominal aortic aneurysm, per 20 MET-hours/week and nonlinear doseresponse analysis.

study quality. Any further studies should investigate the dose-response relationship between different types and intensities of physical activity in relation to risk of abdominal aortic aneurysm, adjust more comprehensively for potential confounding factors, conduct analyses stratified by smoking, and could investigate potential mechanistic pathways that may explain the association between physical activity and lower risk of abdominal aortic aneurysm. In addition, more detailed reporting on the exposure ascertainment and whether it has been validated or not, exclusion of prevalent cases at baseline and/or clear reporting on whether prevalent cases have been excluded at baseline, as well as reporting on loss to follow-up seems to be areas for improving study quality.

The current findings are consistent with recommendations to increase the level of physical activity to reduce the risk of other chronic diseases such as coronary heart disease $\mathrm{e}^{25}$, stroke $^{25}$, type 2 diabetes ${ }^{35}$, several cancers ${ }^{43}$, and other conditions ${ }^{36,37}$, as well as premature mortality ${ }^{44}$, and suggest these benefits perhaps also may extend to abdominal aortic aneurysms. However, further research is needed before these findings can be considered conclusive.

\section{Conclusion}

In conclusion, this meta-analysis suggests that individuals who are physically active have a $30 \%$ reduction in the risk of abdominal aortic aneurysm, however, residual confounding cannot be entirely excluded. Additional studies from geographically diverse regions with better adjustment and stratification for smoking and other potential confounding factors are needed to clarify these associations. Further assessment of the dose-response relationship between different types and intensities of physical activity and risk of abdominal aortic aneurysm and the potential mechanistic pathways that may explain these findings is also needed. 


\begin{tabular}{|c|c|c|c|c|c|}
\hline & \multicolumn{5}{|c|}{ Physical activity and abdominal aortic aneurysm } \\
\hline & $n$ & Relative risk $(95 \% \mathrm{CI})$ & $I^{2}(\%)$ & $P_{h}{ }^{1}$ & $P_{\mathrm{h}}{ }^{2}$ \\
\hline All studies & 9 & $0.70(0.56-0.87)$ & 58.3 & 0.01 & \\
\hline \multicolumn{6}{|l|}{ Sex } \\
\hline Men & 6 & $0.72(0.53-0.98)$ & 61.9 & 0.02 & \multirow{3}{*}{0.7} \\
\hline Women & 0 & & & & \\
\hline Men and women & 3 & $0.66(0.44-0.98)$ & 65.3 & 0.06 & \\
\hline \multicolumn{6}{|l|}{ Outcome type } \\
\hline Incidence & 7 & $0.71(0.55-0.91)$ & 66.3 & 0.007 & \multirow{2}{*}{0.58} \\
\hline Mortality & 2 & $0.62(0.40-0.96)$ & 0 & 0.37 & \\
\hline \multicolumn{6}{|l|}{ Follow-up } \\
\hline$<10$ years & 3 & $0.73(0.57-0.93)$ & 0 & 0.47 & \multirow{2}{*}{0.95} \\
\hline$\geq 10$ years & 6 & $0.69(0.51-0.95)$ & 71.7 & 0.003 & \\
\hline \multicolumn{6}{|l|}{ Geographic location } \\
\hline Europe & 5 & $0.58(0.43-0.79)$ & 48.3 & 0.10 & \multirow{2}{*}{0.13} \\
\hline America & 4 & $0.85(0.64-1.12)$ & 51.6 & 0.10 & \\
\hline \multicolumn{6}{|l|}{ Number of cases } \\
\hline Cases $<150$ & 4 & $0.68(0.39-1.18)$ & 64.7 & 0.04 & \multirow{2}{*}{0.81} \\
\hline Cases $\geq 150$ & 5 & $0.72(0.57-0.91)$ & 59.9 & 0.04 & \\
\hline \multicolumn{6}{|l|}{ Exclusion of prevalent cases at baseline } \\
\hline Yes & 6 & $0.76(0.60-0.96)$ & 61.1 & 0.03 & \multirow{2}{*}{0.24} \\
\hline No & 3 & $0.53(0.35-0.80)$ & 22.4 & 0.28 & \\
\hline \multicolumn{6}{|l|}{ Outcome assessment } \\
\hline Record linkage (hospital, death records) & 8 & $0.71(0.56-0.91)$ & 62.0 & 0.01 & \multirow{2}{*}{0.67} \\
\hline Ultrasound screening & 1 & $0.59(0.36-0.97)$ & & & \\
\hline \multicolumn{6}{|l|}{ Study quality } \\
\hline $0-3$ stars & 0 & & & & \multirow{3}{*}{0.8} \\
\hline $4-6$ stars & 4 & $0.68(0.44-1.05)$ & 66.8 & 0.03 & \\
\hline 7-9 stars & 5 & $0.71(0.54-0.94)$ & 60.0 & 0.04 & \\
\hline Adjustment for confounding factors ${ }^{3}$ & & & & & \\
\hline Age & & & & & \\
\hline Yes & 9 & $0.70(0.56-0.87)$ & 58.3 & 0.01 & NC \\
\hline No & 0 & & & & NC \\
\hline Education & & & & & \\
\hline Yes & 2 & $0.72(0.56-0.93)$ & 0 & 0.34 & 097 \\
\hline No & 7 & $0.70(0.52-0.94)$ & 67.2 & 0.006 & 0.91 \\
\hline Alcohol & & & & & \\
\hline Yes & 3 & $0.85(0.50-1.44)$ & 47.9 & 0.15 & 043 \\
\hline No & 6 & $0.66(0.52-0.86)$ & 66.8 & 0.01 & 0.43 \\
\hline Smoking & & & & & \\
\hline Yes & 5 & $0.76(0.54-1.07)$ & 65.1 & 0.02 & 040 \\
\hline No & 4 & $0.60(0.45-0.80)$ & 57.6 & 0.07 & 0.43 \\
\hline BMI or obesity & & & & & \\
\hline Yes & 4 & $0.86(0.65-1.14)$ & 43.3 & 0.15 & 0 \\
\hline No & 5 & $0.58(0.42-0.80)$ & 60.6 & 0.04 & 0.12 \\
\hline Diabetes mellitus & & & & & \\
\hline Yes & 3 & $0.81(0.61-1.06)$ & 38.7 & 0.20 & 045 \\
\hline No & 6 & $0.65(0.47-0.90)$ & 64.9 & 0.01 & 0.45 \\
\hline Hypertension & & & & & \\
\hline Yes & 2 & $0.80(0.47-1.36)$ & 67.8 & 0.08 & \\
\hline No & 7 & $0.67(0.52-0.86)$ & 59.4 & 0.02 & 0.57 \\
\hline Systolic blood pressure & & & & & \\
\hline Yes & 2 & $0.95(0.56-1.60)$ & 54.2 & 0.14 & בר \\
\hline No & 7 & $0.64(0.50-0.83)$ & 61.1 & 0.02 & 0.23 \\
\hline Diastolic blood pressure & & & & & \\
\hline Yes & 2 & $0.57(0.27-1.17)$ & 77.5 & 0.04 & 051 \\
\hline No & 7 & $0.73(0.57-0.94)$ & 58.3 & 0.03 & 0.51 \\
\hline Continued & & & & & \\
\hline
\end{tabular}




\begin{tabular}{|c|c|c|c|c|c|}
\hline & \multicolumn{5}{|c|}{ Physical activity and abdominal aortic aneurysm } \\
\hline & $n$ & Relative risk $(95 \% \mathrm{CI})$ & $I^{2}(\%)$ & $P_{\mathrm{h}}{ }^{1}$ & $P_{h}^{2}$ \\
\hline \multicolumn{6}{|c|}{ Hypercholesterolemia or serum cholesterol } \\
\hline Yes & 5 & $0.76(0.54-1.07)$ & 65.1 & 0.02 & \multirow{2}{*}{0.49} \\
\hline No & 4 & $0.63(0.46-0.86)$ & 54.5 & 0.09 & \\
\hline \multicolumn{6}{|c|}{ Triglycerides } \\
\hline Yes & 2 & $0.71(0.20-2.55)$ & 86.9 & 0.006 & \multirow{2}{*}{0.96} \\
\hline No & 7 & $0.71(0.58-0.87)$ & 47.4 & 0.08 & \\
\hline
\end{tabular}

Table 2. Subgroup analyses of physical activity and abdominal aortic aneurysm. $n$ denotes the number of studies. ${ }^{1} \mathrm{P}$ for heterogeneity within each subgroup. ${ }^{2} \mathrm{P}$ for heterogeneity between subgroups with metaregression analysis. $B M I$ body mass index, $N C$ not calculable because no studies were present in one of the subgroups.

\section{Material and methods}

Search strategy. Pubmed, and Embase databases were searched up to October 3rd 2020. The full search is described in the Supplementary Text. In addition, we searched the reference lists of the included publications for any additional studies. MOOSE criteria for reporting of meta-analyses were followed ${ }^{45}$.

Study selection and inclusion criteria. We included published retrospective and prospective cohort studies and nested case-control studies within cohorts which reported adjusted estimates of the relative risk (RR) with the 95\% confidence intervals (CIs) for the association between physical activity and the risk of abdominal aortic aneurysm. The excluded studies and exclusion reasons can be found in Supplementary Table 1.

Data extraction. The following data were extracted from the included studies: The first author's name, publication year, country where the study was conducted, study period, sample size, number of cases and participants, exposure and subgroup, RRs and 95\% CIs and variables adjusted for in the analysis. The data were extracted by DA and checked for accuracy by EK.

Statistical methods. The random-effects model by DerSimonian and Laird ${ }^{46}$ was used to calculate summary relative risks (RRs) and 95\% confidence intervals (CIs) of abdominal aortic aneurysm for the highest vs. lowest level of physical activity and per 20 MET-hours per week. The average of the natural logarithm of the RRs was estimated and the RR from each study was weighted using random effects weights. For the linear doseresponse analysis we used the method by Greenland and Longnecker to estimate linear trends and (CIs) across categories of physical activity ${ }^{47}$. For studies that reported physical activity by ranges of activity we calculated the average of the upper and lower cut-off value to obtain an estimate of the midpoint for each category. For studies with open-ended extreme categories we used the width of the adjacent category to estimate an upper and lower cut-off for the highest and lowest category, respectively. Nonlinear dose-response analyses were conducted using restricted cubic splines with knots at 10,50 and 90\% percentiles of the distribution of physical activity, which were combined using multivariate meta-analysis ${ }^{48,49}$.

Because of differences in the way physical activity was reported between studies, conversions to the same scale (MET-hours/week) were made when possible. For one study which reported on walking/bicycling in minutes per day $^{30}$ we used the average of 8 METs for bicycling (equivalent to bicycling at 12-13.9 mph with moderate effort) and 3.3 METs for walking at moderate pace $(3.0 \mathrm{mph}){ }^{50}$, thus we used 5.65 METs as the average intensity for that study. For a second study we used the average of moderate (3-5.9 METs) and most vigorous (6-12 METs) activities to convert minutes/week of activity to METs (although some vigorous activities, e.g. bicycle racing, competitive skating, and ski racing, have METs up to 18 we chose 12 as an upper limit as most of the vigorous activities are in that range and because relatively few participants would be competitive sportsmen ${ }^{50}$ ). Thus we used 6.725 METs as intensity for that study. Another study which used a physical activity index ${ }^{28}$ which had a different scoring of the intensities than the MET values was excluded from the dose-response analysis because it was impossible to convert the reported data to METs. For one study (Cancer Prevention Study 1) which reported physical activity data as none, slight, moderate and heavy we used estimated METs from the Cancer Prevention Study $2^{34}$, but excluded the study in a sensitivity analysis. For one study which reported on aneurysms and peripheral vascular disease combined ${ }^{32}$, the analyses were repeated but restricted to abdominal aortic aneurysm. Two additional studies were only included in the analysis of the highest versus lowest level of physical activity because they had $<3$ categories of physical activity ${ }^{13,29}$. For two studies ${ }^{13,29}$ which compared no physical activity with activity we converted the risk estimates so the comparison became the highest vs. the lowest (consistent with the remaining studies) by inverting the risk estimates and confidence intervals.

Heterogeneity between studies was evaluated using $\mathrm{Q}$ and $\mathrm{I}^{2}$ statistics ${ }^{51}$. $\mathrm{I}^{2}$ measures how much of the heterogeneity is due to between study variation rather than chance. We conducted main analyses (all studies combined) and subgroup analyses stratified by study characteristics to investigate potential sources of heterogeneity. The subgroups included sex, outcome type (incidence vs. mortality), duration of follow-up, geographic location, number of cases, exclusion of prevalent cases at baseline, outcome assessment, study quality and by adjustment for confounding factors (age, education, alcohol, smoking, BMI/obesity, diabetes mellitus, hypertension, systolic 
blood pressure, diastolic blood pressure, hypercholesterolemia or serum cholesterol, and triglycerides. Study quality was assessed using the Newcastle Ottawa scale which rates studies a score from 0 to 9 based on the selection, comparability and outcome assessment of the studies ${ }^{52}$.

Publication bias was assessed using Egger's test ${ }^{53}$ and Begg-Mazumdar's test ${ }^{54}$ and by inspection of funnel plots. The statistical analyses were conducted using the software package Stata, version 13.1 software (StataCorp, Texas, US).

Received: 12 October 2019; Accepted: 26 October 2020

Published online: 18 December 2020

\section{References}

1. Reimerink, J. J., van der Laan, M. J., Koelemay, M. J., Balm, R. \& Legemate, D. A. Systematic review and meta-analysis of populationbased mortality from ruptured abdominal aortic aneurysm. Br. J Surg. 100, 1405-1413 (2013).

2. Verhoeven, E. L. et al. Mortality of ruptured abdominal aortic aneurysm treated with open or endovascular repair. J. Vasc. Surg. 48, 1396-1400 (2008).

3. Sweeting, M. J., Balm, R., Desgranges, P., Ulug, P. \& Powell, J. T. Individual-patient meta-analysis of three randomized trials comparing endovascular versus open repair for ruptured abdominal aortic aneurysm. Br. J Surg. 102, 1229-1239 (2015).

4. Karthikesalingam, A. et al. Mortality from ruptured abdominal aortic aneurysms: clinical lessons from a comparison of outcomes in England and the USA. Lancet 383, 963-969 (2014).

5. GBD 2017 Causes of Death Collaborators. Global, regional, and national age-sex-specific mortality for 282 causes of death in 195 countries and territories, 1980-2017: a systematic analysis for the Global Burden of Disease Study 2017. Lancet 392, 1736-1788 (2018).

6. GBD 2017 Disease and Injury Incidence and Prevalence Collaborators. Global, regional, and national incidence, prevalence, and years lived with disability for 354 diseases and injuries for 195 countries and territories, 1990-2017: a systematic analysis for the Global Burden of Disease Study 2017. Lancet 392, 1789-1858 (2018).

7. Orr, N., Minion, D. \& Bobadilla, J. L. Thoracoabdominal aortic aneurysm repair: current endovascular perspectives. Vasc. Health Risk Manag. 10, 493-505 (2014).

8. Forsdahl, S. H., Singh, K., Solberg, S. \& Jacobsen, B. K. Risk factors for abdominal aortic aneurysms: a 7-year prospective study: the Tromso Study, 1994-2001. Circulation 119, 2202-2208 (2009).

9. Vardulaki, K. A. et al. Incidence among men of asymptomatic abdominal aortic aneurysms: estimates from 500 screen detected cases. J. Med. Screen. 6, 50-54 (1999).

10. Lederle, F. A. et al. Yield of repeated screening for abdominal aortic aneurysm after a 4-year interval. Aneurysm Detection and Management Veterans Affairs Cooperative Study Investigators. Arch. Intern. Med. 160, 1117-1121 (2000).

11. Salem, M. K. et al. Should Asian men be included in abdominal aortic aneurysm screening programmes?. Eur. J. Vasc. Endovasc. Surg. 38, 748-749 (2009).

12. Lederle, F. A. et al. Abdominal aortic aneurysm events in the women's health initiative: cohort study. BMJ 337, a1724 (2008).

13. Tornwall, M. E., Virtamo, J., Haukka, J. K., Albanes, D. \& Huttunen, J. K. Life-style factors and risk for abdominal aortic aneurysm in a cohort of Finnish male smokers. Epidemiology 12, 94-100 (2001).

14. Wong, D. R., Willett, W. C. \& Rimm, E. B. Smoking, hypertension, alcohol consumption, and risk of abdominal aortic aneurysm in men. Am. J. Epidemiol. 165, 838-845 (2007).

15. Jahangir, E. et al. Smoking, sex, risk factors and abdominal aortic aneurysms: a prospective study of 18782 persons aged above 65 years in the Southern Community Cohort Study. J. Epidemiol. Community Health 69, 481-488 (2015).

16. Nordon, I. M., Hinchliffe, R. J., Loftus, I. M. \& Thompson, M. M. Pathophysiology and epidemiology of abdominal aortic aneurysms. Nat. Rev. Cardiol. 8, 92-102 (2011).

17. Kobeissi, E., Hibino, M., Pan, H. \& Aune, D. Blood pressure, hypertension and the risk of abdominal aortic aneurysms: a systematic review and meta-analysis of cohort studies. Eur. J. Epidemiol. 34, 547-555 (2019).

18. Aune, D., Schlesinger, S., Norat, T. \& Riboli, E. Tobacco smoking and the risk of abdominal aortic aneurysm: a systematic review and meta-analysis of prospective studies. Sci. Rep. 8, 14786 (2018).

19. Aune, D., Schlesinger, S., Norat, T. \& Riboli, E. Diabetes mellitus and the risk of abdominal aortic aneurysm: a systematic review and meta-analysis of prospective studies. J. Diabetes Complicat. 32, 1169-1174 (2018).

20. Cornelissen, V. A. \& Smart, N. A. Exercise training for blood pressure: a systematic review and meta-analysis. J. Am. Heart Assoc. 2, e004473 (2013).

21. Barengo, N. C. et al. Low physical activity as a predictor for antihypertensive drug treatment in 25-64-year-old populations in eastern and south-western Finland. J. Hypertens. 23, 293-299 (2005).

22. Hu, G. et al. Relationship of physical activity and body mass index to the risk of hypertension: a prospective study in Finland. Hypertension 43, 25-30 (2004).

23. Williams, P. T. A cohort study of incident hypertension in relation to changes in vigorous physical activity in men and women. $J$. Hypertens. 26, 1085-1093 (2008).

24. Pereira, M. A. et al. Physical activity and incident hypertension in black and white adults: the Atherosclerosis Risk in Communities Study. Prev. Med. 28, 304-312 (1999).

25. Kyu, H. H. et al. Physical activity and risk of breast cancer, colon cancer, diabetes, ischemic heart disease, and ischemic stroke events: systematic review and dose-response meta-analysis for the Global Burden of Disease Study 2013. BMJ 354, i3857 (2016).

26. Pandey, A. et al. Dose-response relationship between physical activity and risk of heart failure: a meta-analysis. Circulation 132, 1786-1794 (2015).

27. Hammond, E. C. \& Garfinkel, L. Coronary heart disease, stroke, and aortic aneurysm. Factors in the etiology. Arch. Environ. Health 19, 167-182 (1969).

28. Goldberg, R. J. et al. Lifestyle and biologic factors associated with atherosclerotic disease in middle-aged men. 20-year findings from the Honolulu Heart Program. Arch. Intern. Med. 155, 686-694 (1995).

29. Lindblad, B., Borner, G. \& Gottsater, A. Factors associated with development of large abdominal aortic aneurysm in middle-aged men. Eur. J. Vasc. Endovasc. Surg. 30, 346-352 (2005).

30. Stackelberg, O. et al. Lifestyle and risk of screening-detected abdominal aortic aneurysm in men. J. Am. Heart Assoc. 6, e004725 (2017).

31. Nordkvist, S., Sonestedt, E. \& Acosta, S. Adherence to diet recommendations and risk of abdominal aortic aneurysm in the Malmo Diet and Cancer Study. Sci. Rep. 8, 2017 (2018).

32. Hamer, M., O’Donovan, G. \& Stamatakis, E. Association between physical activity and sub-types of cardiovascular disease death causes in a general population cohort. Eur. J. Epidemiol. 34, 483-487 (2019). 
33. Oyenuga, A. O., Folsom, A. R., Lutsey, P. L. \& Tang, W. Association of Life's Simple 7 with reduced clinically manifest abdominal aortic aneurysm: the ARIC study. Vasc. Med. 1358863 X19829226 (2019).

34. Patel, A. V. et al. The relationship between physical activity, obesity, and lung cancer risk by smoking status in a large prospective cohort of US adults. Cancer Causes Control 28, 1357-1368 (2017).

35. Aune, D., Norat, T., Leitzmann, M., Tonstad, S. \& Vatten, L. J. Physical activity and the risk of type 2 diabetes: a systematic review and dose-response meta-analysis. Eur. J Epidemiol. 30, 529-542 (2015).

36. Aune, D., Leitzmann, M. \& Vatten, L. J. Physical activity and the risk of gallbladder disease: a systematic review and meta-analysis of cohort studies. J. Phys. Act. Health 13, 788-795 (2016).

37. Aune, D., Sen, A., Henriksen, T., Saugstad, O. D. \& Tonstad, S. Physical activity and the risk of gestational diabetes mellitus: a systematic review and dose-response meta-analysis of epidemiological studies. Eur. J Epidemiol. 31, 967-997 (2016).

38. Kelley, G. A., Kelley, K. S. \& Tran, Z. V. Aerobic exercise and lipids and lipoproteins in women: a meta-analysis of randomized controlled trials. J. Womens Health (Larchmt) 13, 1148-1164 (2004).

39. Kelley, G. A. \& Kelley, K. S. Aerobic exercise and lipids and lipoproteins in men: a meta-analysis of randomized controlled trials. J Mens. Health Gend. 3, 61-70 (2006).

40. Sofi, F., Capalbo, A., Cesari, F., Abbate, R. \& Gensini, G. F. Physical activity during leisure time and primary prevention of coronary heart disease: an updated meta-analysis of cohort studies. Eur. J. Cardiovasc. Prev. Rehabil. 15, 247-257 (2008).

41. Iribarren, C. et al. Traditional and novel risk factors for clinically diagnosed abdominal aortic aneurysm: the Kaiser multiphasic health checkup cohort study. Ann. Epidemiol. 17, 669-678 (2007).

42. Gibson, C. et al. Mild aerobic exercise blocks elastin fiber fragmentation and aortic dilatation in a mouse model of Marfan syndrome associated aortic aneurysm. J. Appl. Physiol. 123, 147-160 (2017).

43. Moore, S. C. et al. Association of leisure-time physical activity with risk of 26 types of cancer in 1.44 million adults. JAMA Intern. Med. 176, 816-825 (2016).

44. Samitz, G., Egger, M. \& Zwahlen, M. Domains of physical activity and all-cause mortality: systematic review and dose-response meta-analysis of cohort studies. Int. J. Epidemiol. 40, 1382-1400 (2011).

45. Stroup, D. F. et al. Meta-analysis of observational studies in epidemiology: a proposal for reporting. Meta-analysis of observational studies in epidemiology (MOOSE) group. JAMA 283, 2008-2012 (2000).

46. DerSimonian, R. \& Laird, N. Meta-analysis in clinical trials. Control Clin. Trials 7, 177-188 (1986).

47. Greenland, S. \& Longnecker, M. P. Methods for trend estimation from summarized dose-response data, with applications to metaanalysis. Am. J. Epidemiol. 135, 1301-1309 (1992).

48. Jackson, D., White, I. R. \& Thompson, S. G. Extending DerSimonian and Laird's methodology to perform multivariate random effects meta-analyses. Stat. Med. 29, 1282-1297 (2010).

49. Orsini, N., Li, R., Wolk, A., Khudyakov, P. \& Spiegelman, D. Meta-analysis for linear and nonlinear dose-response relations: examples, an evaluation of approximations, and software. Am. J. Epidemiol. 175, 66-73 (2012).

50. Ainsworth, B. E. et al. Compendium of physical activities: an update of activity codes and MET intensities. Med. Sci. Sports Exerc. 32, S498-S504 (2000).

51. Higgins, J. P. \& Thompson, S. G. Quantifying heterogeneity in a meta-analysis. Stat. Med. 21, 1539-1558 (2002).

52. Wells, G. et al. The Newcastle-Ottawa Scale (NOS) for assessing the quality of nonrandomised studies in meta-analyses. https:// www.ohri.ca/programs/clinical_epidemiology/oxford.asp, Accessed August 8th 2018.

53. Egger, M., Davey, S. G., Schneider, M. \& Minder, C. Bias in meta-analysis detected by a simple, graphical test. BMJ 315, 629-634 (1997).

54. Begg, C. B. \& Mazumdar, M. Operating characteristics of a rank correlation test for publication bias. Biometrics 50, 1088-1101 (1994).

\section{Acknowledgements}

This work has been supported by funding from Raagholtsstiftelsen and the South-East Regional Health Authority of Norway.

\section{Author contributions}

D.A. conducted the literature search, did the data extraction, analyses and wrote the first draft of the paper. D.A., A.S. contributed to the literature screening and E.K. checked the data extraction for accuracy. DA., A.S, E.K, M.H., T.N, E.R. interpreted the data, revised the subsequent drafts for important intellectual content, read and approved the final manuscript.

\section{Competing interests}

The authors declare no competing interests.

\section{Additional information}

Supplementary information is available for this paper at https://doi.org/10.1038/s41598-020-76306-9.

Correspondence and requests for materials should be addressed to D.A.

Reprints and permissions information is available at www.nature.com/reprints.

Publisher's note Springer Nature remains neutral with regard to jurisdictional claims in published maps and institutional affiliations.

(c) (i) Open Access This article is licensed under a Creative Commons Attribution 4.0 International License, which permits use, sharing, adaptation, distribution and reproduction in any medium or format, as long as you give appropriate credit to the original author(s) and the source, provide a link to the Creative Commons licence, and indicate if changes were made. The images or other third party material in this article are included in the article's Creative Commons licence, unless indicated otherwise in a credit line to the material. If material is not included in the article's Creative Commons licence and your intended use is not permitted by statutory regulation or exceeds the permitted use, you will need to obtain permission directly from the copyright holder. To view a copy of this licence, visit http://creativecommons.org/licenses/by/4.0/.

(C) The Author(s) 2020 